\title{
Effect of Lansoprazole on the Control of the Intragastric pH in a Patient with Short Bowel Syndrome
}

\author{
Masato Yoshioka, Tasuku Watanabe, Masatake Iida, Yasuhiko Nakagawa, Hiroshi Uchinami, \\ Go Watanabe, Yuki Abe, Fumitaka Yagi, Naohiro Yokoyama and Yuzo Yamamoto
}

\begin{abstract}
:
A 40-year-old man suffered from short bowel syndrome. Since a large amount of intestinal output and watery diarrhea hampered his quality of life, we tried to control the intestinal output by reducing the secretion of gastric acid with lansoprazole. Because the small intestine was only $10 \mathrm{~cm}$ in length and effective absorption of oral lansoprazole was doubtful, we monitored his intragastric $\mathrm{pH}$ for 24 hours and confirmed that the holding time above $\mathrm{pH} 3.0$ was 14.5 hours $(60.4 \%)$. He spent his home life eating porridge during the day, and receiving total parenteral nutrition of $1,100 \mathrm{~mL} /$ day at night while taking lansoprazole as an oral tablet (30 mg) once a day.
\end{abstract}

Key words: short bowel syndrome (SBS), proton pump inhibitor (PPI), lansoprazole, pH monitor, gastric juice

(Intern Med 58: 1723-1726, 2019)

(DOI: 10.2169/internalmedicine.2221-18)

\section{Introduction}

Short bowel syndrome (SBS) refers to the malabsorption caused by the functional or physical loss of the small intestine. In adult patients, physical loss usually results from extensive intestinal resection for Crohn's disease, thrombosis or embolism of mesenteric vasculature, volvulus, malignancy, trauma, etc. $(1,2)$. Management of SBS aims to supply nutrition and fluid. In patients with SBS, diarrhea usually occurs due to accelerated intestinal transit, increased gastrointestinal secretion, intestinal bacterial overgrowth, and malabsorption of fats and bile salts (3). Among these factors, fat malabsorption is strongly related to the increase in gastric acid. Therefore, to alleviate the fat malabsorption, controlling the gastric acid production is important.

Proton pump inhibitors (PPIs) and H2-blockers are known to be effective in reducing the intestinal output in SBS patients. However, whether or not oral anti-gastric acid agents can be absorbed when the small intestine is extremely short is unclear. No report has measured the change in the intragastric $\mathrm{pH}$ after the oral administration of PPI or H2blockers in an SBS patient.
We herein report a patient with SBS and an extremely short jejunum $(10 \mathrm{~cm}$ in length) in whom the intragastric $\mathrm{pH}$ was well controlled by the oral administration of the PPI lansoprazole (LPZ).

\section{Case Report}

A 40-year-old man with acute aortic dissection from the ascending aorta to the bilateral common iliac artery (Stanford type A) with occlusion of the right external iliac artery underwent thoracic aortic repair using a J Graft open stent graft $^{\circledR}$ (Japan Lifeline, Tokyo, Japan) and femorofemoral crossover bypass surgery using a Gelsoft ${ }^{\circledR}$ (Terumo, Tokyo, Japan) graft. Eight days after surgery, he developed severe abdominal pain with signs of rebound tenderness. Computed tomography (CT) showed perfusion failure of the superior mesenteric artery. Emergency laparotomy revealed massive intestinal necrosis from the jejunum to the middle of transverse colon necessitating subtotal resection of the small intestine and right hemicolectomy. Only $10 \mathrm{~cm}$ of the jejunum from the ligament of Treitz remained, and jejunostomy and transverse colostomy were constructed.

The patient survived, but his intestinal output from the je-

Department of Gastroenterological Surgery, Akita University Graduate School of Medicine, Japan

Received: October 2, 2018; Accepted: November 26, 2018; Advance Publication by J-STAGE: February 25, 2019

Correspondence to Dr. Masato Yoshioka, masato@gipc.akita-u.ac.jp 


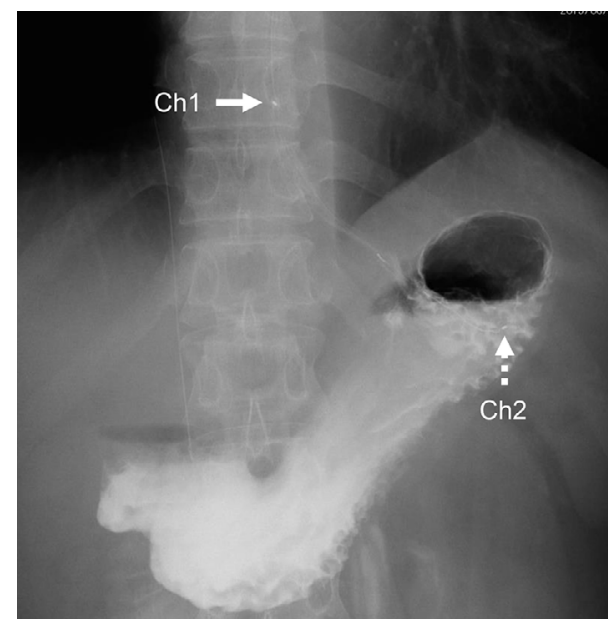

Figure 1. An X-ray photogram under an upper gastrointestinal series shows two $\mathrm{pH}$ sensors. Channel 1 is placed at $15 \mathrm{~cm}$ from esophagogastric junction and channel 2 is placed in the stomach. Ch1: channel 1 (white arrow), Ch2: channel 2 (white dotted arrow).

junostomy amounted to about 2,000 mL/day. Because infusion of $3,500 \mathrm{~mL} /$ day was necessary, we administered the H2-blocker famotidine intravenously at a dose of $20 \mathrm{mg}$ twice a day. His intestinal output apparently decreased to $1,400 \mathrm{~mL} /$ day after a few days, and the infusion volume was reduced to $2,700 \mathrm{~mL}$ /day. However, there was no change in his condition even at two months after surgery.

To reduce his intestinal output, we closed the jejunostomy by jejuno-transverse colon anastomosis. After this surgery, we started LPZ intravenously at a dose of $30 \mathrm{mg}$ twice a day in place of famotidine. Although the frequency of watery stool was 8 to 9 times a day at the closure of stoma, it gradually decreased to 6 times a day. The volume of infusion was able to be reduced to $2,000 \mathrm{~mL} /$ day. He received total parenteral nutrition (TPN) $(1,680 \mathrm{kcal})$ and ate rice porridge once during the day. With the goal of allowing the patient to live at home and to provide relief from TPN in the future, we tried to change LPZ administration from intravenous to an oral form. However, we were unsure whether or not a jejunum of only $10 \mathrm{~cm}$ in length could absorb orally taken LPZ. Therefore, we had to test whether or not oral LPZ worked.

We used orally disintegrating (OD) tablets $(30 \mathrm{mg}$ ) and measured the intragastric $\mathrm{pH}$ continuously for 24 hours under fasting condition using a Digitrapper ${ }^{\mathrm{TM}} \mathrm{pH} 400$ monitoring system (Medtronic, Minneapolis, USA). Before the test, we had to eliminate the effects of LPZ used in the preceding days. For this purpose, we instated a 4-day 'wash out' period for LPZ because Bell et al. reported that the intragastric $\mathrm{pH}$ had returned to the control level by the fourth day postdosing of LPZ-giving an estimated half-life of 22 hours-in healthy volunteers (4). However, we worried that abruptly stopping intravenous LPZ for 4 days would jeopardize the patient's condition, so we temporarily switched intravenous LPZ to intravenous famotidine for the first 2 days, at a dose

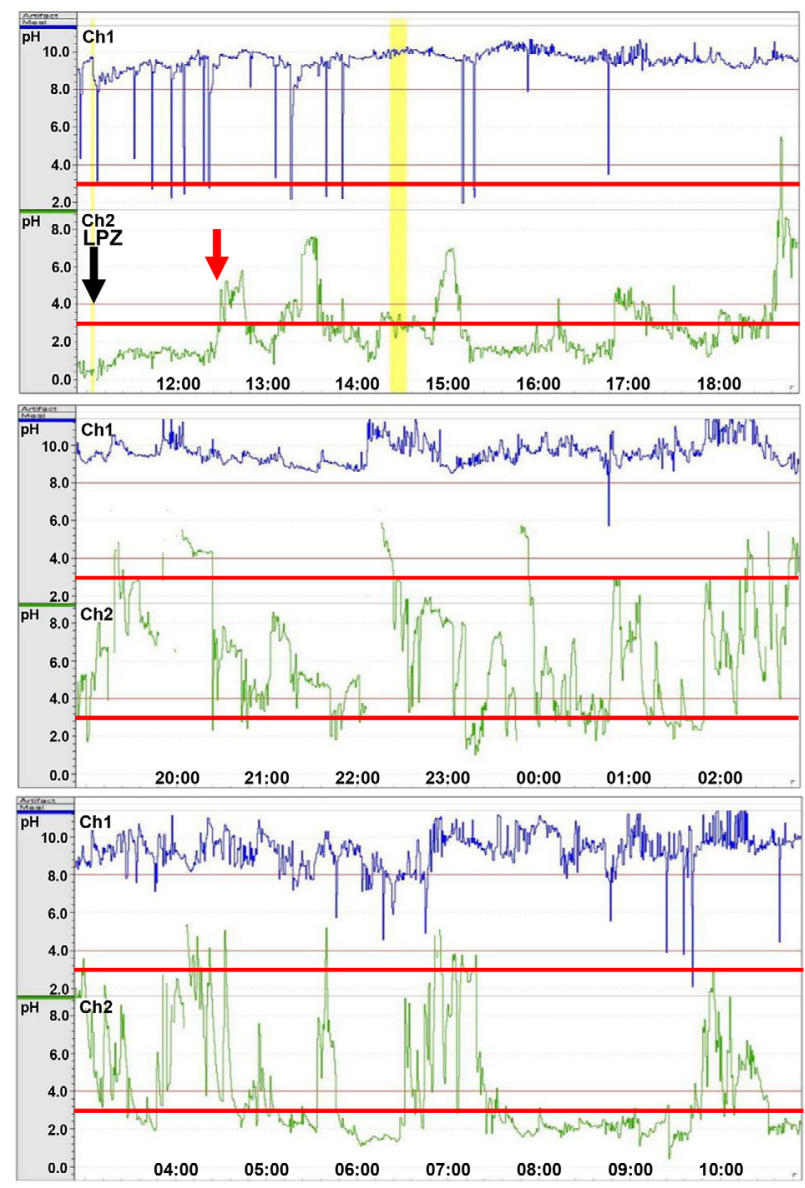

Figure 2. A 24-hour pH trendgram. Measurement was started at 11: 00 (black arrow) with the administration of a $30 \mathrm{mg}$ lansoprazole (LPZ) orally disintegrating tablet. Ninety minutes after taking LPZ, the $\mathrm{pH}$ of the gastric juice increased above 4.0 (red arrow). The blue line graph shows the $\mathrm{pH}$ at channel (Ch) 1 in the esophagus, and the green line graph shows the $\mathrm{pH}$ of $\mathrm{Ch} 2$ in the stomach. The horizontal bold red line shows pH 3.0. Yellow bands indicate the time of events. The first yellow band indicates the start of the test. The second broad yellow band at 14: 30 was recorded by accidentally touching the switch.

of $20 \mathrm{mg}$ twice a day. Famotidine was then also discontinued for another 2 days because the Interview Form of famotidine showed that the blood concentration after intravenous administration became null within 24 hours (5), and the inhibitory effect on gastric acid secretion was positively correlated with the blood concentration (6). During this two-day discontinuation of all anti-gastric acid agents, the frequency of watery stool increased to eight times a day.

According to the dosage regimen of oral lansoprazole, a single $30 \mathrm{mg}$ LPZ OD tablet was administered, and its effects were then monitored for 24 hours. Electrodes of $\mathrm{pH}$ sensors were placed nasally. Fig. 1 shows the positions of the two sensors. Channel (Ch) 1 , in the esophagus, is a sensor for monitoring gastroesophageal reflux. $\mathrm{Ch} 2$, in the stomach, is a sensor for monitoring the $\mathrm{pH}$ of the gastric juice. Fig. 2 shows the 24-hour $\mathrm{pH}$ trendgram of the present case. We paid attention to the two points of $\mathrm{pH}$ level-3.0 
and 4.0-in the stomach, because a $\mathrm{pH}$ below 3.0 indicated that gastric acid was retained, which induced diarrhea, while a $\mathrm{pH}$ above 4.0 implied the prevention of reflux esophagitis.

Before administering LPZ, we monitored the baseline intragastric $\mathrm{pH}$ for 30 minutes on the display screen. During this period, the intragastric $\mathrm{pH}$ was roughly below 1 , as the last 10-minute portion was recorded in the first part of Fig. 2. This observation was compatible with the baseline $\mathrm{pH}$ level in the control group without medication in published reports (7-10). LPZ was administered at 11:00 AM. Even after taking LPZ, the intragastric $\mathrm{pH}$ remained below 2 for about 90 minutes. It then steeply increased to above 4.0 at 90 minutes but then showed a slow fluctuation of rises and falls across pH 3.0 for about 6 hours in the daytime, with a substantial increase above $\mathrm{pH} 3.0$ achieved later in the evening. This 24-hour measurement showed that the holding time above $\mathrm{pH} 3.0$ was 14.5 hours $(60.4 \%)$, while that above $\mathrm{pH} 4.0$ was 11.3 hours (47.1\%). Gastroesophageal reflux did not occur, and Ch1 continuously showed a $\mathrm{pH}$ above 8.0.

While taking an LPZ OD tablet $(30 \mathrm{mg}$ ) once a day, the patient ate rice porridge during the day, and received TPN of $1,100 \mathrm{~mL} /$ day $(840 \mathrm{kcal})$ through a central venous access port at night. With this life cycle, he was shifted to outpatient treatment. Over the 16 months after the closure of the stoma, the patient was able to live at home with a defecation frequency of 4 times a day until he unfortunately died of an aortic graft infection and mediastinitis due to methicillinresistant Staphylococcus aureus.

\section{Discussion}

More than half of intestinal resection patients experience an increase in gastric acid levels after surgery. This phenomenon is thought to be caused by the loss of endogeneous intestinal inhibitors of gastric acid secretion (11), such as cholecystokinin, secretin, and neurotensin (12), or the hypersecretion of gastrin (13). PPIs are typical first-line agents for treatment as they achieve the near-complete suppression of gastric acid secretion in healthy persons (14). H2blockers and PPIs are known to reduce the intestinal fluid in patients with SBS as well. Jacobsen et al. reported that 8 patients (small intestine 60-220 cm) with jejunostomy were treated with the intravenous H2-blocker cimetidine (15), and the intestinal output was reduced in all patients. Aly et al. reported that 10 patients with diarrhea after extensive resection of the small intestine were treated with oral cimetidine, and all showed a decrease in intestinal output (16). Indeed, famotidine decreased the intestinal output from jejunostomy from $2,000 \mathrm{~mL} /$ day to $1,400 \mathrm{~mL} /$ day in our patient. Regarding PPIs, Nightingale et al. reported that omeprazole reduced the intestinal output in SBS patients (17). In their report, the intestinal output decreased due to the oral administration of omeprazole in a patient with a remaining jejunum length of only $25 \mathrm{~cm}$. In addition, the effect of oral omeprazole was equivalent to its intravenous infusion, and omepra- zole showed equivalent efficacy to ranitidine. However, while they noted a decrease in the intestinal output, they failed to demonstrate the absorption of omeprazole by measuring the serum concentration or the intragastric $\mathrm{pH}$. No report has yet described the elevation of intragastric $\mathrm{pH}$ following the oral administration of a PPI in SBS patients.

The Interview Form of LPZ published by Takeda Pharmaceutical Company Limited showed that the serum concentration of LPZ increases from 30 minutes after the oral administration of LPZ and peaks at 2 hours (18). Regarding the time delay until the substantial elevation of intragastric $\mathrm{pH}$ above 3.0, Suzuki et al. reported that the mean time to reach a $\mathrm{pH}$ above 3.0 was $3.75 \pm 0.48$ hours in healthy volunteers (19). Given these data in healthy people, the elevation of $\mathrm{pH}$ above 4.0 within 90 minutes after dosing seems unlikely. In this regard, the observation of fluctuations across $\mathrm{pH} 3.0$ until 18:00 might imply the insufficient concentration of LPZ and thus an incomplete or unstable absorption of LPZ due to the short intestinal length. It might be correct to conclude that about 8 hours was necessary until a sufficient effect was obtained in this patient. However, this would not be very meaningful in clinical practice, as unlike single dosing, repeated daily dosing would result in a cumulative effect (4).

When patients took LPZ under a fasting condition, the mean $\mathrm{pH}$ holding time above 3.0 was 21.3 hours (89\%), although these data were obtained following the administration of LPZ in capsule form (18). Unfortunately, there have been no time studies of the intragastric $\mathrm{pH}$ with LPZ OD tablets. However, the pharmacokinetics $\left(\mathrm{C}_{\max }\right.$ and $\left.\mathrm{AUC}_{0-24}\right)$ of the LPZ capsule were the same as those of the LPZ OD tablet. In the present patient, the $\mathrm{pH}$ holding time above 3.0 was 14.5 hours $(60.4 \%)$. Given this result and the similar findings of the previous case report (17), even if the remaining length of the jejunum is extremely short after extensive resection, an LPZ OD tablet can be absorbed with about two-thirds the efficacy in a healthy person with a full-length small intestine. Although we did not measure the serum level of LPZ, measuring the intragastric $\mathrm{pH}$ as a surrogate marker proved the effective absorption of LPZ. Oral LPZ by OD tablet was useful for controlling intragastric $\mathrm{pH}$ and was thereby able to ameliorate the cumbersome excess of the intestinal output in an SBS patient.

The limitation of this case report is that we were unable to confirm the absorption of LPZ from the $10-\mathrm{cm}$ jejunum because we did not measure the serum concentration of LPZ during $\mathrm{pH}$ monitoring. Since such extreme SBS patients are rarely encountered, we strongly encourage measuring the serum LPZ concentration in the next such patient.

In conclusion, the absorption of LPZ from an OD tablet in a patient with a jejunum length of only $10 \mathrm{~cm}$ was shown to be effective by measuring the intragastric $\mathrm{pH}$. The use of an LPZ OD tablet is recommended for controlling intestinal output in SBS patients.

The authors state that they have no Conflict of Interest (COI). 


\section{References}

1. Jeppesen PB. Spectrum of short bowel syndrome in adults: intestinal insufficiency to intestinal failure. JPEN J Parenter Enteral Nutr 38 (1 Suppl): 8S-13S, 2014.

2. Buchman AL, Scolapio J, Fryer J. AGA technical review on short bowel syndrome and intestinal transplantation. Gastroenterology 124: 1111-1134, 2003.

3. Kumpf VJ. Parmacologic management of diarrhea in patients with short bowel syndrome. JPEN J Parenter Enteral Nutr 38 (1 Suppl): 38S-44S, 2014.

4. Bell N, Karol MD, Sachs G, Greski-Rose P, Jennings DE, Hunt RH. Duration of effect of lansoprazole on gastric $\mathrm{pH}$ and acid secretion in normal male volunteers. Aliment Pharmacol Ther 15: 105-113, 2001.

5. LTL Pharma Company Limited. Interview Form of $\mathrm{H} 2$ receptor antagonist, famotidine. 14th ed. 2018.

6. Miwa M, Tani N, Miwa T. Inhibition of gastric secretion by a new H2-antagonist, YM-11170 in healthy subjects. Int J Clin Pharmacol Ther Toxicol 22: 214-217, 1984.

7. Shirai N, Furuta T, Xiao F, et al. Comparison of lansoprazole and famotidine for gastric acid inhibition during the daytime and night-time in different CYP2C19 genotype groups. Aliment Pharmacol Ther 16: 837-846, 2002.

8. Hongo M, Ohara S, Hirasawa Y, Abe S, Asaki S, Toyota T. Effect of lansoprazole on intragastric $\mathrm{pH}$. Comparison between morning and evening dosing. Dig Dis Sci 37: 882-890, 1992.

9. Geus WP, Mulder PG, Nicolai JJ, Van den Boomgaard DM, Lamers CB. Acid-inhibitory effects of omeprazole and lansoprazole in Helicobacter pylori-negative healthy subjects. Aliment Pharmacol Ther 12: 329-335, 1998.

10. Shimatani $T$, Inoue $M$, Kuroiwa $T$, et al. Acid-suppressive effects of rabeprazole, omeprazole, and lansoprazole at reduced and standard doses: a crossover comparative study in homozygous exten- sive metabolizers of cytochrome P450 2C19. Clin Pharmacol Ther 79: 144-152, 2006.

11. Thompson JS, Rochling FA, Weseman RA, Mercer DF. Current management of short bowel syndrome. Curr Probl Surg 49: 52$115,2012$.

12. Schubert ML. Hormonal regulation of gastric acid secretion. Curr Gastroenterol Rep 10: 523-527, 2008.

13. Straus E, Gerson CD, Yalow RS. Hypersecretion of gastrin associated with the short bowel syndrome. Gastroenterology 66: 175180, 1974.

14. Howden CW, Forrest JA, Reid JL. Effects of single and repeated doses of omeprazole on gastric acid and pepsin secretion in man. Gut 25: 707-710, 1984.

15. Jacobsen O, Ladefoged K, Stage JG, Jarnum S. Effect of cimetidine on jejunostomy effluents in patients with severe short bowel syndrome. Scand J Gastroenterol 21: 824-828, 1986.

16. Aly A, Bárány F, Kollberg B, Monsén U, Wisén O, Johansson C. Effect of an H2-receptor blocking agent on diarrhoeas after extensive small bowel resection in Crohn's disease. Acta Med Scand 207: 119-122, 1980.

17. Nightingale JM, Walker ER, Farthing MJ, Lennard-Jones JE. Effect of omeprazole on intestinal output in the short bowel syndrome. Aliment Pharmacol Ther 5: 405-412, 1991.

18. Takeda Pharmaceutical Company Limited. Interview Form of proton pump inhibitor, lansoprazole. 18th ed. 2017.

19. Suzuki T, Yamaguchi T, Okada T, et al. Four-day continuous gastric $\mathrm{pH}$ monitoring following anti-acid secretory drug administration: cross-over test to assess the early effects. Aliment Pharmacol Ther 27: 66-71, 2008.

The Internal Medicine is an Open Access journal distributed under the Creative Commons Attribution-NonCommercial-NoDerivatives 4.0 International License. To view the details of this license, please visit (https://creativecommons.org/licenses/ by-nc-nd/4.0/).

(C) 2019 The Japanese Society of Internal Medicine Intern Med 58: 1723-1726, 2019 